\title{
Reprodução de Pseudis minuta (Anura, Hylidae) no sul do Brasil
}

\author{
Janaine Melchiors', Marcos Di-Bernardo ${ }^{1,2}$, Glaucia Maria Funk Pontes ${ }^{1,2}$, Roberto \\ Baptista de Oliveira ${ }^{1,2}$, Mirco Solé ${ }^{3}$ e Axel Kwet ${ }^{4}$ \\ 1 Faculdade de Biociências, Pontifícia Universidade Católica do Rio Grande do Sul (PUCRS). Av. Ipiranga, 6681, \\ 90619-900, Porto Alegre, RS, Brasil. E-mail: jmelchi@pucrs.br. \\ 2 Museu de Ciências e Tecnologia, PUCRS. \\ 3 Zoologisches Institut der Universität Tübingen, Auf der Morgenstelle 28, D-72076 Tübingen, Alemanha. \\ 4 Staatliches Museum für Naturkunde, Rosenstein 1, D-70191, Stuttgart, Alemanha.
}

\begin{abstract}
Reproduction of Pseudis minuta (Anura, Hylidae) in southern Brazil. This study was based on individuals of Pseudis minuta captured or observed in the municipality of Candiota, Campanha region of the state of Rio Grande do Sul, southern Brazil. Sampling occurred along ten non-consecutive months in 2000, 2001 and 2002. The reproductive phases were characterized based on the gonadal development stage of 39 males and 50 females, and on the observation, in nature, of the seasonal distribution of calling males, occurrence of amplectant pairs, and presence of larvae, juveniles and adults. Calls were recorded from August to April, amplectant pairs from October to February (except January), tadpoles from October to March (except February), and juveniles in all the sampled months (except January). Reproductive activity was not observed in late fall and early winter, even though females with post-vitellogenic oocytes and males with spermatozoa in the seminiferous tubules were recorded in all the seasons. Mature females were statistically larger and heavier than mature males. The smallest female with post-vitellogenic oocytes had $32.0 \mathrm{~mm}$ of snout-vent length, and the smallest male with spermatozoa in its seminiferous tubules had $20.6 \mathrm{~mm}$. The number of post-vitellogenic oocytes was directly proportional to the mass and to the snout-vent length of females, and the length of testis was directly proportional to the snout-vent length and to the mass of males.
\end{abstract}

Keywords: Anura, Hylidae, Pseudinae, Pseudis minuta, reproduction, southern Brazil. Palavras-chave: Anura, Hylidae, Pseudinae, Pseudis minuta, reprodução, sul do Brasil.

\section{Introdução}

Tradicionalmente incluídos na família Pseudidae Savage e Carvalho, 1953 (e.g.

Recebido em 4 de março de 2004.

Aceito em 4 de agosto de 2004.

Distribuído em 30 de setembro de 2004.
Gallardo 1961, de Sá e Lavilla 1997, Caramaschi e Cruz 1998, Kwet e Di-Bernardo 1999, Kwet 2000, 2001), os anuros dos gêneros Lysapsus e Pseudis foram considerados por Duellman (2001) como pertencentes à família Hylidae, subfamília Pseudinae. A posição filogenética desse grupo ainda é controversa (Brandão et al. 2003), e seguimos Duellman 
(2001) em função da metodologia cladística adotada em seu estudo.

A subfamília Pseudinae Fitzinger, 1843 é composta por espécies exclusivamente aquáticas, que ocorrem na América do Sul a leste dos Andes, desde a Venezuela até o nordeste da Argentina e Uruguai (Kwet e Di-Bernardo 1999). Compreende as espécies Lysapsus caraya Gallardo, 1964, L. laevis (Parker, 1935), L. limellus Cope, 1862, Pseudis bolbodactyla Lutz, 1925, P. cardosoi Kwet, 2000, P. fusca Garman, 1883, P. minuta Günther, 1858, P. paradoxa (Linnaeus, 1758) e $P$. tocantins Caramaschi \& Cruz, 1998 (Kwet 2000). Os pseudíneos vivem usualmente em ambientes lênticos, em poças permanentes ou temporárias com vegetação flutuante. Como adaptação à vida aquática, apresentam os artelhos unidos por membranas interdigitais bem desenvolvidas e olhos dispostos na região dorsal da cabeça (Achaval e Olmos 1997). Grande parte dos estudos realizados com Pseudis minuta (Figura 1) baseou-se em animais procedentes do Uruguai (Langone 1995 “1994”; Lavilla e de Sá 1999) e Argentina (Cei 1949, Barrio 1970, Gallardo 1987, Basso 1990). Pseudis minuta apresenta estação reprodutiva prolongada (Langone 1995 “1994”) e, assim como ocorre com a maioria das espécies de anuros, no período reprodutivo os machos congregam-se em determinadas áreas com a finalidade de atrair fêmeas e se acasalar.

O presente trabalho objetivou a caracterização das fases reprodutivas de $P$. minuta no sul do Brasil a partir da análise do estágio de desenvolvimento gonadal de machos e fêmeas e da observação, na natureza, da distribuição sazonal de machos em vocalização, ocorrência de amplexos e presença de formas larvais, juvenis e adultas.

\section{Material e Métodos}

A área de estudo está situada no município de Candiota, sudoeste do estado do Rio Grande do Sul, no quadrilátero de coordenadas $31^{\circ} 25^{\prime}-$

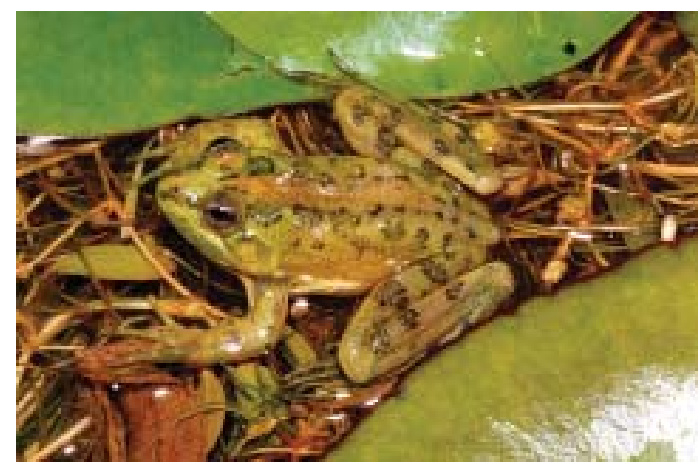

Figura 1 - Espécime de Pseudis minuta procedente de Candiota, RS.

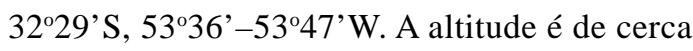
de 290 m acima do nível do mar, e a vegetação é não-florestal, do tipo Estepe. Nessa região, não há déficit pluviométrico, chovendo usualmente o ano inteiro (Instituto Brasileiro de Geografia e Estatística 1986). As temperaturas médias mensais são típicas de áreas com clima subtropical a temperado, havendo estações bem definidas, com temperaturas baixas no inverno e elevadas no verão (Figura 2).

Os dados aqui apresentados resultaram de observações e coletas realizadas em outubro e

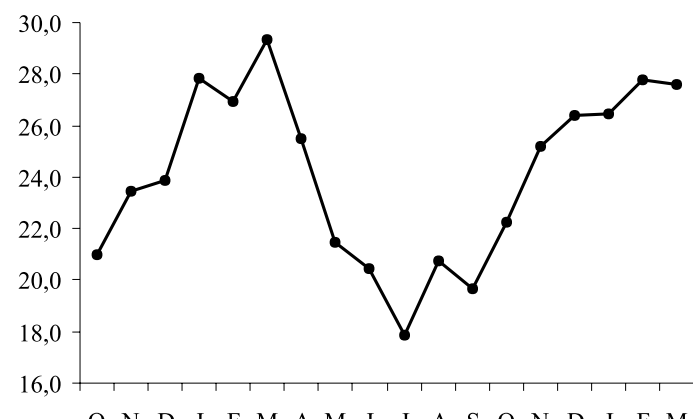

Figura 2 - Temperaturas mensais médias entre outubro de 2000 e março de 2002 no município de Candiota (RS). Fonte: Estação Meteorológica da Usina Termelétrica Presidente Médici. 
dezembro de 2000, janeiro, abril, junho, agosto, outubro e novembro de 2001 e fevereiro e março de 2002. Indivíduos juvenis e adultos foram capturados manualmente no interior de banhados, sem distinção de sexo ou tamanho, entre 21:00 e 01:00 h. Girinos foram coletados com puçás e acondicionados em potes plásticos com água. Os juvenis e os adultos foram sacrificados por imersão em álcool $40 \%$ e fixados em formalina $5 \%$, e os girinos foram sacrificados e fixados diretamente por imersão em formalina $5 \%$. Os espécimes coletados foram depositados no acervo do Museu de Ciências e Tecnologia da Pontifícia Universidade Católica do Rio Grande do Sul (MCP).

O comprimento rostro-cloacal (CRC, em $\mathrm{mm}$ ) e a massa (g) foram obtidos para cada espécime capturado, após a fixação. Para a remoção e a análise das gônadas, os espécimes foram secionados na região ventral. $\mathrm{O}$ comprimento dos testículos foi aferido com o auxílio de ocular micrométrica acoplada a microscópio estereoscópico. Para a análise histológica do ciclo espermatogênico, os testículos de 39 machos foram incluídos em parafina, cortados em fatias de $5 \mu \mathrm{m}$ de espessura (Montero e Pisanó 1992) e corados com hematoxilinafloxina. Os testículos foram processados íntegros, incluindo-se as regiões cranial, medial e caudal (Hermosilla et al. 1983). Foram considerados maduros os machos com saco vocal desenvolvido e evidente ou com espermatozóides nos túbulos seminíferos. Os ovários foram analisados quanto ao estágio de maturação, seguindo-se a terminologia proposta por Hermosilla et al. (1986) e Valdéz-Toledo e Pisanó (1980), com modificações. Foram considerados os seguintes estágios de desenvolvimento vitelogênico: a) ovócitos pré-vitelogênicos: translúcidos, sem acúmulo de vitelo no citoplasma; b) ovócitos em vitelogênese primária: coloração leitosa, sem pigmentação; início da vitelogênese; c) ovócitos pósvitelogênicos: maiores que os anteriores, com hemisférios diferenciados (pólo animal marromescuro e pólo vegetativo leitoso, de coloração marfim). O número de ovócitos pós-vitelogênicos foi contado, e o diâmetro dos dez maiores ovócitos, medido. Para facilitar a contagem, os ovócitos foram individualizados por imersão em solução de hipoclorito de sódio a $10 \%$. O tempo de imersão foi dependente do tamanho do ovário, sendo em média de 20 minutos. Foram consideradas maduras as fêmeas com ovócitos pós-vitelogênicos.

A fecundidade foi estimada com base no complemento ovariano (número de ovócitos pós-vitelogênicos no ovário) e no Fator de Tamanho Ovariano (FTO; Duellman e Crump 1974), que relaciona o número de ovócitos pósvitelogênicos e seu tamanho com o CRC segundo a fórmula FTO $=\mathrm{CO} \times \mathrm{DO} / \mathrm{LHC}$, onde CO é a média do número de ovócitos pósvitelogênicos, DO é o diâmetro médio dos ovócitos pós-vitelogênicos e LHC é a média do CRC.

O ciclo ovariano das fêmeas foi analisado considerando-se a variação anual no tamanho e pigmentação dos folículos ovarianos. Foram considerados maduros todos os ovócitos com pólo animal e vegetal diferenciados.

O período reprodutivo foi determinado com base na atividade de vocalização dos machos e na presença de casais em amplexo e girinos (Prado e Uetanabaro 2000).

O dimorfismo sexual em tamanho (CRC e massa) de machos e fêmeas maduros foi avaliado pelo teste $t$ de Student (normalidade testada com Kolmogorov-Smirnov). As relações entre o tamanho (comprimento e massa) dos testículos e o CRC, o número de ovócitos pósvitelogênicos e a massa e o número de ovócitos pós-vitelogênicos e o CRC foram calculadas por meio de análise de regressão linear.

\section{Resultados}

Vocalizações foram registradas de agosto a abril, amplexos, de outubro a fevereiro (exceto janeiro), girinos, de outubro a março (exceto fevereiro), e juvenis, em todos os meses estudados (exceto janeiro) (Tabela 1). 
Tabela 1 - Distribuição temporal das diferentes fases reprodutivas e etárias de Pseudis minuta em Candiota (RS). Não estão representados os meses nos quais não foram feitas coletas.

\begin{tabular}{lccccccccc}
\hline & JAN & FEV & MAR & ABR & JUN & AGO & OUT & NOV & DEZ \\
\hline Vocalização & $\mathrm{X}$ & $\mathrm{X}$ & $\mathrm{X}$ & $\mathrm{X}$ & & $\mathrm{X}$ & $\mathrm{X}$ & $\mathrm{X}$ & $\mathrm{X}$ \\
Amplexo & & $\mathrm{X}$ & & & & & $\mathrm{X}$ & $\mathrm{X}$ & $\mathrm{X}$ \\
Girinos & $\mathrm{X}$ & & $\mathrm{X}$ & & & & $\mathrm{X}$ & $\mathrm{X}$ & $\mathrm{X}$ \\
Juvenis & & $\mathrm{X}$ & $\mathrm{X}$ & $\mathrm{X}$ & $\mathrm{X}$ & $\mathrm{X}$ & $\mathrm{X}$ & $\mathrm{X}$ & $\mathrm{X}$ \\
\hline
\end{tabular}

O CRC das fêmeas maduras variou entre 32,0 e $51,1 \mathrm{~mm}(\overline{\mathrm{x}}=42,2 ; \mathrm{DP}=4,2 ; \mathrm{N}=50)$ e a massa, entre 4,4 e 20,8 g $(\bar{x}=12,0 ; D P=3,5$; $\mathrm{N}=50$ ) (Figura 3). O CRC dos machos maduros variou entre 20,6 e 36,9 mm ( $\bar{x}=30,8$; $D P=4,2$; $\mathrm{N}=39)$ e a massa, entre 1,3 e $6,8 \mathrm{~g}(\overline{\mathrm{x}}=4,6$; $\mathrm{DP}=1,7 ; \mathrm{N}=39$ ) (Figura 3). Fêmeas maduras apresentaram CRC médio e massa média significativamente maior que machos maduros $(t=$ 12,3 ; $\mathrm{P}<0,001$ e $t=11,6$; $\mathrm{P}<0,001$, respectivamente).

Os ovários de fêmeas maduras são estruturas pareadas, multilobuladas e saculares. Ovócitos em vários estágios de desenvolvimento puderam ser observados por transparência, através da parede externa. No estágio avançado de maturação, os ovários ocupam quase toda a cavidade abdominal. Todos os estágios do desenvolvimento ovariano puderam ser observados em todos os meses amostrados, mas

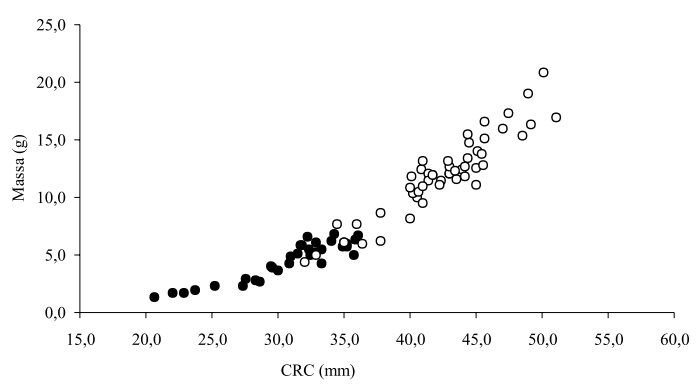

Figura 3 - Relação entre o comprimento rostro-cloacal e a massa de machos maduros $(\bullet)$ e fêmeas maduras (o) de Pseudis minuta procedentes de Candiota (RS). apenas em outubro, novembro, fevereiro, março e abril foram encontrados ovócitos pós-vitelogênicos no interior dos ovidutos. Não foram visualizados corpos negros nem ovócitos em auxocitose.

O FTO foi de 10,5 (CO=337; $\mathrm{DO}=1,32 \mathrm{~mm}$; $\mathrm{LHC}=42,2 \mathrm{~mm})$. O número de ovócitos pósvitelogênicos variou de 101 a $744(\overline{\mathrm{x}}=351,1$; $\mathrm{DP}=158,4 ; \mathrm{N}=50$ ), e foi diretamente proporcional à massa $\left(\mathrm{R}^{2}=0,3242 ; \mathrm{F}=23,0 ; \mathrm{P}<0,001\right.$; $\mathrm{N}=50)$ (Figura 4) e ao CRC $\left(\mathrm{R}^{2}=0,2339\right.$; $\mathrm{F}=14,7 ; \mathrm{P}<0,001 ; \mathrm{N}=50$ ) (Figura 5). Não foi constatada relação entre o diâmetro dos ovócitos pós-vitelogênicos e o CRC das fêmeas (Correlação de Pearson, $\mathrm{R}=0,162 ; \mathrm{P}=0,26$; $\mathrm{N}=50$ ).

Não foram encontradas diferenças histológicas nos elementos seminíferos das três zonas testiculares. No interior dos túbulos, foram encontradas células germinativas em diferentes

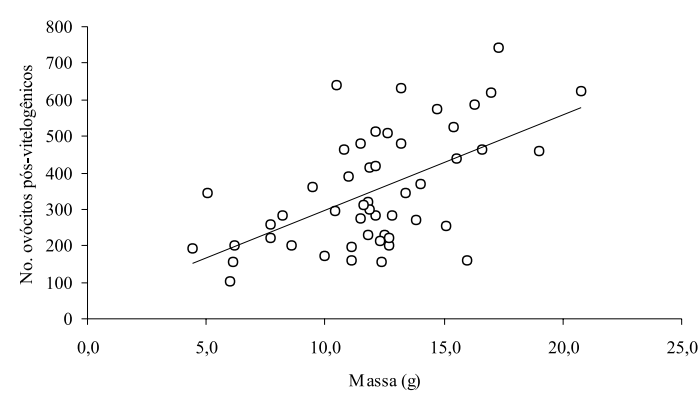

Figura 4 - Relação entre o número de ovócitos pósvitelogênicos e a massa de fêmeas de Pseudis minuta procedentes de Candiota (RS). 


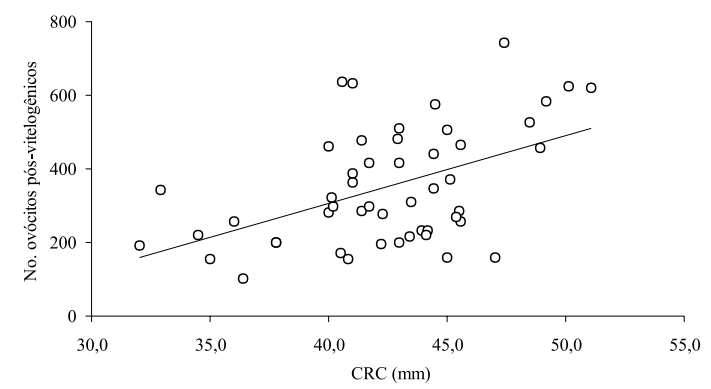

Figura 5 - Relação entre o número de ovócitos pósvitelogênicos e o comprimento rostro-cloacal (CRC) de fêmeas de Pseudis minuta procedentes de Candiota (RS).

estágios de maturação. As células em estágios mais avançados de maturação foram encontradas com maior freqüência no lúmen tubular. As células germinativas e os espermatozóides variaram de estágio ou posição dentro dos túbulos seminíferos ao longo das estações do ano: verão (dezembro, janeiro e fevereiro; $\mathrm{N}=7$ ) - espermatogênese ativa, com células germinativas em diferentes fases da progênie espermática, geralmente dispostas perifericamente; grande concentração de espermatozóides na luz do túbulos seminíferos; outono (março e abril; $\mathrm{N}=7$ ) - células germinativas e espermatozóides em pequena quantidade; espermatozóides distribuídos aleatoriamente nos túbulos seminíferos; inverno (junho e agosto; $\mathrm{N}=11$ ) e primavera (outubro e novembro; $\mathrm{N}=14$ ) - células germinativas em diferentes estágios de desenvolvimento; grande concentração de espermatozóides distribuídos aleatoriamente nos túbulos seminíferos.

O comprimento dos testículos foi diretamente proporcional ao $\mathrm{CRC}\left(\mathrm{R}^{2}=0,5011\right.$; $\mathrm{F}=151,7 ; \mathrm{P}<0,001 ; \mathrm{N}=153$ ) (Figura 6) e à massa $\left(\mathrm{R}^{2}=0,4223 ; \mathrm{F}=108,2 ; \mathrm{P}<0,001 ; \mathrm{N}=149\right)$ (Figura 7).

\section{Discussão}

Pseudis minuta apresenta o modo reprodutivo mais generalizado e filogeneticamente mais primitivo entre os anuros (Duellman e Trueb 1994, Basso 1990), em que os ovos e os girinos,

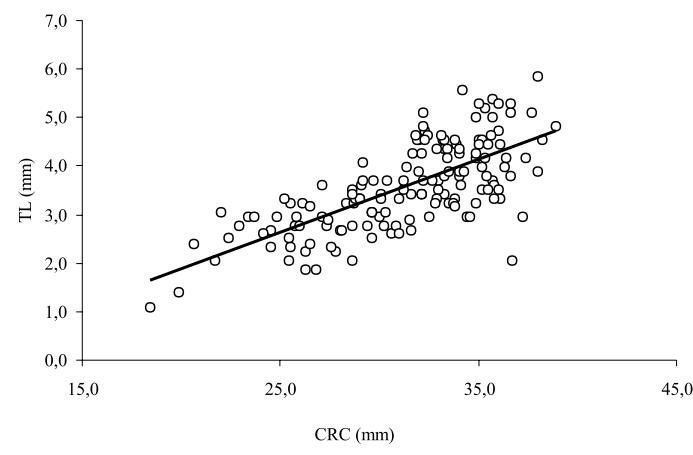

Figura 6 - Relação entre o comprimento do testículo (TL) e o comprimento rostro-cloacal (CRC) de machos de Pseudis minuta procedentes de Candiota (RS).

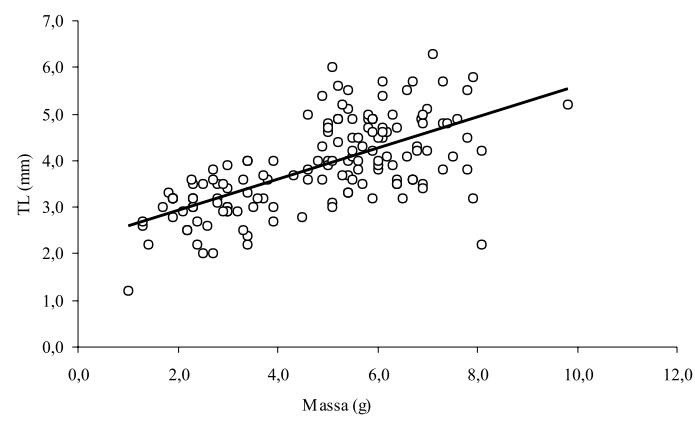

Figura 7 - Relação entre o comprimento do testículo (TL) e a massa de machos de Pseudis minuta procedentes de Candiota (RS).

respectivamente, são depositados e se desenvolvem em ambientes lênticos (Salthe e Duellman 1973, Crump 1974).

As fêmeas são maiores que os machos conspecíficos em 90\% das espécies de anuros (Shine 1979). No Equador, Crump (1974) encontrou fêmeas maiores que machos conspecíficos em 59 das 61 espécies examinadas e, no Planalto das Araucárias do Rio Grande do Sul, Kwet (2001) verificou que as fêmeas de todas as espécies de hilídeos eram maiores que os machos conspecíficos. Diversos podem ser os fatores que determinam a ocorrência de dimorfismo sexual em tamanho (Stamps 1995). Uma das hipóteses está relacionada à capacidade de fêmeas maiores produzirem ovos ou desovas 
maiores (maior fecundidade) em relação a fêmeas conspecíficas menores (Crump 1974, Woolbright 1989, Lang 1995). Além disso, fêmeas maiores apresentam grande probabilidade de produzir mais de uma desova por temporada reprodutiva (Howard 1978, Telford e Dyson 1990). Contudo, nem sempre a fecundidade é diretamente proporcional ao comprimento do corpo, podendo estar mais relacionada ao volume corporal e ao tipo de investimento reprodutivo utilizado pela espécie (Halliday e Tejedo 1995, Lang 1995, Bonnet et al. 2003). Lavilla e Rougés (1992) denominaram a forma reprodutiva de Pseudis minuta de extrínseca cíclica. Essa forma caracteriza-se por reprodução estacional, mas com a presença de espermatozóides e óvulos maduros durante todo o ano. Neste caso, a distribuição temporal das diferentes etapas reprodutivas e etárias (e.g. épocas de vocalização, amplexos, ocorrência de girinos e juvenis na população) pode melhor delinear o ciclo reprodutivo e melhor caracterizar a biologia reprodutiva da espécie quando comparada com a morfologia e o estágio de desenvolvimento das estruturas gonadais.

Na região de Candiota, a reprodução de $P$. minuta é provavelmente condicionada pela temperatura ambiental, havendo períodos de inatividade invernal (Guix 1996), mas a hipótese de uma potencial atividade reprodutiva continuada não pode ser descartada. A ocorrência de machos com espermatozóides e fêmeas com ovócitos pós-vitelogênicos nos meses mais frios, quando as vocalizações foram pouco freqüentes e quando não foram registrados amplexos nem girinos na população, pode representar a potencialidade da espécie se reproduzir mesmo durante o inverno, em períodos curtos com temperaturas mais amenas (Gallardo 1964, Guix 1996). Na população estudada, os girinos provavelmente completaram a metamorfose entre dezembro e março (supondo um período de desenvolvimento completo de dois meses e meio a quatro meses; Langone 1995 “1994”), e os juvenis metamorfoseados tardiamente não dispuseram de muitos meses com temperaturas elevadas para se desenvolver, permanecendo como juvenis até a próxima estação quente.

Assim como ocorre na Argentina (Basso 1990), em Candiota, P. minuta poderia estar maximizando sua eficácia biológica invertendo sua energia reprodutiva, ou seja, produzindo ovos em pequena quantidade, mas de grande tamanho e com maior conteúdo calórico. Ovos grandes têm um efeito importante sobre a taxa de sobrevivência dos recém-nascidos, especialmente por ocasião do nascimento e nos primeiros dias subseqüentes, quando as possibilidades de predação dos filhotes são maiores (Cunnington e Brooks 2000). Contudo, quase nada se conhece a esse respeito para $P$. minuta, uma espécie abundante e de fácil observação nas áreas baixas do sul do Brasil e um excelente modelo para o teste de hipóteses dessa natureza.

\section{Agradecimentos}

A Cynthia Prado, Marília Hartmann, Adrian Garda e Raúl Maneyro por sugestões e auxílio na obtenção de artigos. A John Measey, Márcio Borges-Martins e Tatiana Miranda pelo auxílio durante as coletas. Ao Instituto Brasileiro do Meio Ambiente e dos Recursos Naturais Renováveis (IBAMA) pela concessão de licenças para coleta dos anuros (números 171/99 DIFAS e 015/201 - RAN). A Betina Blochtein pelas facilidades junto ao Laboratório de Histologia da PUCRS. Os trabalhos de campo foram financiados com recursos do PADCT/ GTM (chamada GTM-01/97-02/05) conferidos a MD. MD é bolsista do Conselho Nacional de Desenvolvimento Científico e Tecnológico (CNPq, processo 300.164/00-9).

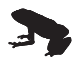




\section{Referências Bibliográficas}

Achaval, F. e A. Olmos. 1997. Anfibios y Reptiles del Uruguay. Montevideo, Barreiro y Ramos S. A. 128 pp.

Barrio, A. 1970. Caracteres del canto nupcial de los Pséudidos (Amphibia, Anura). Physis 29: 511-515.

Basso, N. G. 1990. Estrategias adaptativas en una comunidad subtropical de anuros. Cuadernos de Herpetología (Serie Monografías) 1: 3-70.

Bonnet, X., R. Shine, O. Lourdais e G. Naulleau. 2003. Measures of reproductive allometry are sensitive to sampling bias. Functional Ecology 17: 39-49.

Brandão, R. A., A. Garda, V. Braz e B. Fonseca. 2003. Observations on the ecology of Pseudis bolbodactyla (Anura, Pseudidae) in central Brazil. Phyllomedusa 2: 3-8.

Caramaschi, U. e C. A. G. Cruz. 1998. Notas taxonômicas sobre Pseudis fusca Garman e P. bolbodactyla A. Lutz, com descrição de uma nova espécie correlata (Anura: Pseudidae). Revista Brasileira de Zoologia 15: 929944.

Cei, J. M. 1949. El predominio de la espermatogénesis anual continua em batracios Chaqueños. Acta Zoológica Lilloana 7: 527-550.

Crump, M. L. 1974. Reproductive strategies in a tropical anuran community. Miscellaneous Publications of the Museum of Natural History of the University of Kansas 61: 4-69.

Cunnington, D. C. e R. J. Brooks. 2000. Optimal egg size theory: Does predation by fish affect egg size in Ambystoma maculatum? Journal of Herpetology 34: 46-53.

de Sá, R. O. e E. O. Lavilla. 1997. The tadpole of Pseudis minuta (Anura: Pseudidae), an apparent case of heterochrony. Amphibia-Reptilia 18: 229-240.

Duellman, W. E. e M. L. Crump. 1974. Speciation in frogs of the Hyla parviceps group in the Upper Amazon Basin. Occasional Papers of the Museum of Natural History of the University of Kansas 23: 1-40.

Duellman, W. E. 2001. The Hylid Frogs of Middle America. Ithaca, NY. Society for the Study of Amphibians and Reptiles. 1159 pp.

Duellman, W. e L. Trueb. 1994. Biology of Amphibians. Baltimore and London. The Johns Hopkins University Press. $670 \mathrm{pp}$

Gallardo, J. M. 1961. On the species of Pseudidae (Amphibia, Anura). Bulletin of the Museum of Comparative Zoology 125: 111-134.

Gallardo, J. M. 1964. Una nueva forma de Pseudidae (Amphibia, Anura) y algunas consideraciones sobre las especies argentinas de esta familia. Acta Zoológica Lilloana 20: 193-209.
Gallardo, J. M. 1987. Anfibios Argentinos. Buenos Aires. Biblioteca Mosaico. 98 pp.

Guix, J. C. 1996. Actividad invernal de anuros en tres sierras del sudeste de Brasil. Boletín de la Asociación Herpetológica Española 7: 31-34.

Halliday T. R. e M. Tejedo. 1995. Intrasexual selection and alternative mating behaviour. Pp. 419-468 in $\mathrm{H}$. Heatwole e B. K. Sullivan (eds.), Amphibian Biology. Chipping Norton. Surrey Beatty \& Sons.

Hermosilla, I. B., A. P. Urbina e J. Cabrera. 1983. Espermatogénesis en la Rana Chilena Caudiverbera caudiverbera (Linné) (Anura, Leptodactylidae). Boletín de la Sociedad de Biología de Concepción 54: 103-115.

Hermosilla, I. B., L. Coloma, G. Weigerth, E. T. Reyest e V. Gomezo. 1986. Caracterización del ovario de la "Rana Chilena” Caudiverbera caudiverbera (Linné, 1758) (Anura Leptodactylidae). Boletín de la Sociedad de Biología de Concepción 57: 37-57.

Howard, R. D. 1978. The evolution of mating strategies in bullfrogs Rana catesbeiana. Evolution 32: 850-871.

Instituto Brasileiro de Geografia e Estatística. 1986. Geografia do Brasil. Rio de Janeiro. Secretaria de Planejamento da Presidência e Instituto de Geografia e Estatística (IBGE), v.2. 419 pp.

Kwet, A. 2000. The genus Pseudis (Anura: Pseudidae) in Rio Grande do Sul, southern Brazil, with description of a new species. Amphibia-Reptilia 21: 39-55.

Kwet, A. 2001. Frösche im brasilianischen Araukarienwald - Anurengemeinschaft des Araukarienwaldes von Rio Grande do Sul: Diversität, Reproduktion und Ressourcenaufteilung. Münster. Natur und Tier-Verlag. 192 pp.

Kwet, A. e M. Di-Bernardo. 1999. Pró-Mata: Anfibios. Amphibien. Amphibians. Porto Alegre. EDIPUCRS. 107 pp.

Lang, C. 1995. Size-fecundity relationships among streambreeding hylid frogs. Herpetological Natural History 3: 193-197.

Langone, J. A. 1995 “1994”. Ranas y Sapos del Uruguay (Reconocimiento y Aspectos Biológicos). Museo Dámaso Antonio Larranaga, Serie de Divulgación 5: $1-123$.

Lavilla, E. O. e R. O. de Sá. 1999. Estructura del condrocráneo y esqueleto visceral de larvas de Pseudis minutus (Anura, Pseudidae). Alytes 16: 139-147.

Lavilla, E. O. e M. Rougés. 1992. Reproducción y desarrollo de anuros Argentinos. Asociación Herpetológica Argentina, Serie de Divulgación 5: 1-66.

Montero, R. e A. Pisanó. 1992. El ciclo espermatogénico anual de Hyla pulchella andina: un análisis numérico. Acta Zoológica Lilloana 41: 173-180. 
Prado, C. P. A. e M. Uetanabaro. 2000. Reproductive biology of Lysapsus limellus Cope, 1862 (Anura, Pseudidae) in the Pantanal, Brazil. Zoocriaderos 3: 25-30.

Salthe, S. e W. E. Duellman. 1973. Quantitative constraints associated with reproductive mode in anurans. Pp. 229-249 in J. L. Vial (ed.), Evolutionary Biology of the Anurans - contemporary research in major problems. Columbia. University of Missouri Press.

Shine, R. 1979. Sexual selection and sexual dimorphism in the Amphibia. Copeia 1979: 297-306.
Stamps, J. A. 1995. Using growth-based models to study behavioral factors affecting sexual size dimorphism. Herpetological Monographs 9: 75-87.

Telford, S. R. e M. L. Dyson. 1990. The effect of rainfall on interclutch interval in painted reed frogs (Hyperolius marmoratus). Copeia 1990: 644-648.

Valdez-Toledo, C. L. e A. Pisanó. 1980. Fases ovogenéticas en Bufo arenarum. Reproducción 4: 315-330.

Woolbright, L. L. 1989. Sexual dimorphism in Eleutherodactylus coqui: selection pressures and growth rates. Herpetologica 45: 68-74. 